\title{
La planificación en los Procesos de Admisión Estudiantil
}

\author{
Planning Student Admission Processes
}

- LENIN URDANETA

leninesneider86@gmail.com

Código ORCID: 0000-0002-8338-9423

Universidad del Zulia, Venezuela

Artículo recibido en octubre 2019 / Arbitrado en noviembre 2019 / Publicado en enero 2020

Resumen Se presenta este artículo donde se analizó la planificación en los procesos de admisión estudiantil. Apoyada en una investigación descriptiva, bajo un diseño no experimental, transeccional, de campo. La población objeto de estudio, estuvo conformada por 51 sujetos informantes de las tres instituciones universitarias públicas de la Costa Oriental del Lago, a quienes se les aplicó un instrumento tipo cuestionario, conformado por 18 ítems con escala de frecuencia, se utilizó el juicio de expertos para la validez del mismo y el coeficiente Alfa Cronbach para la confiabilidad, cuyo valor fue de 0,97. El análisis de los datos se realizó mediante la estadística descriptiva, a través del estudio de la media aritmética. Se concluyó una alta aplicación de la planificación como un proceso a través del cual se establecen los cursos de acción adecuados para alcanzar los objetivos trazados en los procesos de admisión analizados.

Palabras clave: Admisión estudiantil; cursos de acción; objetivos; planificación; procesos

\begin{abstract}
This article was presented where the planning in the processes of student admission was analyzed. Supported in a descriptive investigation, under a non-experimental, transectional, field design. The population under study, consisted of 51 informant subjects of the three public university institutions of the East Coast of the Lake, to whom a questionnaire-type instrument was applied, consisting of 18 items with frequency scale, the expert judgment was used for its validity and the Alfa Cronbach coefficient for reliability, whose value was 0.97 . The data analysis was performed using descriptive statistics, through the study of arithmetic mean. A high application of planning concluded as a process through which the appropriate courses of action are established to achieve the objectives set in the admissions processes analyzed.
\end{abstract}

Keywords: Student admission; courses of action; objectives; planning; processes 


\section{INTRODUCCIÓN}

La elección de una carrera a nivel universitario debe constituir una decisión de trascendencia para la autorrealización como profesional y personal de todo joven. En este sentido, el estudiante una vez cubierto sus estudios de segundo nivel y debidamente orientado, probablemente elegirá la opción más acorde a sus características personales, a la realidad socioeconómica y ocupacional así como a los requerimientos exigidos para el ingreso a las instituciones universitarias del país.

De manera que los procesos de admisión estudiantil permiten la planificación de la admisión como proceso sistémico de la política matricular de las instituciones universitarias, concebida en tres componentes fundamentales: Admisión-información, tamaño de la matrícula y la permanencia estudiantil.

Ahora bien, como todo sistema social afectado por la postmodernidad, el comercio, los mercados, y por ende las organizaciones, deben afrontar los cambios de la sociedad industrial de una manera simétrica y efectiva.

En este sentido, las organizaciones, inclusive las instituciones universitarias, se han visto en la necesidad de establecer un proceso de gestión para poder abordarla con una visión clara de crecimiento, por lo que un eficiente planificación de las actividades, proporciona el buen desempeño en las organizaciones, involucrando al personal de la empresa para lograr la efectividad en el producto final, sea este un bien o un servicio, cumpliendo las expectativas del cliente.

Desde esta perspectiva, es importante la planificación, que según Chiavenato (2007) es concebida como un proceso mediante la cual los decisores en una organización, analizan y procesan información de su entorno interno y externo, evaluando las diferentes situaciones vinculadas a la ejecutoria organizacional para prever y decidir sobre la direccionalidad futura.

Ahora bien, el proceso de planificación en el contexto universitario, específicamente en los de admisión estudiantil, las instituciones universitarias deben realizar esfuerzos mancomunados que les permitan llevar a cabo transformaciones, introduciendo adelantos e innovaciones que respondan de manera eficaz a los progresos y cambios que definen esta época, asumiendo este reto como estrategia para consolidar las universidades.

En el caso específico de las instituciones universitarias públicas de la Costa Oriental del Lago, el proceso de admisión se centra en los fundamentos legales de la educación universitaria venezolana, cuyo fin principal es la inserción de jóvenes según sea el Plan de Crecimiento Matricular de cada institución.

Es de hacer notar, que a través de un diagnóstico previo, realizado por el investigador, se pudo evidenciar en cuanto a los procesos de gestión logística la improvisación en el momento de establecer el cronograma de asignación de documentos, falta de personal de apoyo que oriente a los nuevos ingresos, carencia de tecnología de vanguardia disponible para los aspirantes de escasos recursos que requieren procesar su inscripción vía web, la asignación masiva en algunas carreras o programas académicos y la poca en otras.

Por ello se hace necesario realizar un análisis del proceso de planificación en los procesos de admisión estudiantil de las instituciones universitarias públicas de la 
Costa Oriental del Lago, a fin de garantizarles a los nuevos aspirantes un mejor servicio en el momento de ingresar.

\section{Planificación}

Storner y Wankel (2005) indican que la planificación es el proceso para establecer metas y un curso de acción adecuado para alcanzarlas. Implica que los administradores piensen con antelación en sus metas y acciones, y que basen sus actos en algún método, plan o lógica, y no en suposiciones. Armonizando con Hurtado (2008) quien expresa que la planeación fija los objetivos y la misión, examina alternativas, establece los recursos necesarios y finalmente crea estrategias para alcanzar los objetivos.

Cabe considerar, por otra parte lo expuesto por Hellriegel y col. (2005) quien plantea que el proceso de planificación comprende una secuencia de tareas principales, como lo son: la elaboración de la misión, visión, objetivos, realizar el diagnóstico externo y el diagnóstico interno. Recalcan en afirmar que estas no necesariamente tienen que realizarse en una secuencia estricta y que incluso se pueden saltar tareas.

El autor de esta investigación consideró que los elementos sustanciales para ser aplicados en la etapa de planificación de la gestión logística en los procesos de admisión estudiantil, son los pautados por los autores antes mencionados, quienes parten de la elaboración de la visión, la misión, los objetivos y metas.

Desde la perspectiva más general para Sople (2007), la planificación es aquella parte de la gestión logística que se encarga de definir los recursos necesarios para cada función de la organización así como las áreas de atención y de mejoras para lograr los objetivos generales de la empresa, lo cual indudablemente repercutirá en el rendimiento de la organización y en el grado de satisfacción de los clientes.

Los aspectos relacionados con la planificación logística incluyen la toma de decisiones sobre las áreas en que se deben invertir los recursos, si se deben expandir o diversificar las operaciones, también conlleva decisiones acerca de cuáles son las estrategias alternativas que proporcionarán mayores beneficios, las cuales determinaran las ventajas competitivas a largo plazo.

De lo antes expuesto, para el autor llevar a cabo la planificación de la gestión logística en los procesos de admisión estudiantil de las instituciones universitarias públicas de la Costa Oriental del Lago, contempla los aspectos del análisis interno y externo de la logística, creación de la misión, visión, así como la formulación de objetivos y estrategias logísticas.

\section{Análisis interno}

Según expresa David (2008), este comienza por una auditoría que se basa en la participación de todos los gerentes $y$ empleados de la empresa para determinar las fortalezas y las debilidades de la misma, a través de la recolección y asimilación de información sobre las operaciones de dirección, logística, mercadotecnia, finanzas y contabilidad, producción y operaciones, investigación $y$ desarrollo y sistemas de información de la gerencia de la empresa, de tal manera que las fortalezas y las debilidades más importantes de la empresa se determinen de manera colectiva. 
Asimismo, Serna (2008) considera que el análisis interno de la empresa se base en un perfil de capacidad interna de la organización $(\mathrm{PCl})$, el cual es un medio para evaluar las fortalezas y debilidades de la compañía en relación con las oportunidades y amenazas que le presenta el medio externo, involucrando todos los factores que afectan la operación corporativa de la empresa.

Entre los factores que inciden en el análisis interno de la empresa, se tiene la capacidad directiva, la cual se refiere a todas aquellas fortalezas o debilidades que tengan que ver con el proceso administrativo, específicamente en las áreas de planeación, dirección, toma de decisiones, coordinación, comunicación y control.

De igual manera, se debe tomar en cuenta la capacidad competitiva, que son todos los aspectos relacionados con el área comercial, como calidad del producto, exclusividad, portafolio de productos, participación en el mercado, canales de distribución, cubrimiento, investigación y desarrollo, precios, publicidad, lealtad de los clientes, calidad en el senvicio al cliente, entre otros.

Otro factor importante para el análisis es la capacidad financiera, esta incluye todos los aspectos relacionados con las fortalezas o debilidades financieras de la compañía como: deuda o capital, disponibilidad de línea de crédito, capacidad de endeudamiento, margen financiero, rentabilidad, liquidez, rotación de cartera, rotación de inventarios, estabilidad de costos, elasticidad de la demanda y otros índices financieros que se consideren importantes para la organización y el área de análisis.
A su vez es importante conocer la capacidad tecnológica, aquí se incluyen todos los aspectos relacionados con el proceso de producción en las empresas industriales y con la infraestructura y los procesos en las empresas de senvicio. Por tanto, involucra, entre otras: infraestructura tecnológica, exclusividad de los procesos de producción, normalización de los procesos, ubicación física, acceso a servicios públicos, facilidades físicas, intensidad en el uso de mano de obra, patentes, nivel tecnológico, flexibilidad en la producción, disponibilidad de programas de computación, procedimientos administrativos, procedimientos técnicos entre otros.

Finalmente, la capacidad de talento humano es sin duda un factor primordial en el análisis interno de la empresa. Esta capacidad se refiere a todas las fortalezas y debilidades relacionadas con el personal e incluye: nivel académico, experiencia técnica, estabilidad, rotación, absentismo, nivel de remuneración, capacitación, programas de desarrollo, motivación y pertenencia.

A juicio del investigador, para lograr el análisis interno de la organización, es importante efectuar auditorías periódicas que consistan en evaluar todas las capacidades internas de la empresa, con la finalidad de conocer la situación de la organización respecto a cada uno de los factores descritos anteriormente.

\section{Análisis externo}

Opinan Thompson y Strickland (2007) que el análisis externo comprende la revisión de las variables que rodean el ambiente organizacional, incluyendo oportunidades y amenazas. Las oportunidades son aquellos 
factores que resultan positivos, favorables, explotables, que se deben descubrir en el entorno en el que actúa la empresa, y que permiten obtener ventajas competitivas. Dentro de las oportunidades deben considerarse el comportamiento de la empresa en el mercado, la competitividad, así como las tendencias políticas, sociales, económicas y tecnológicas.

Agregan los autores que las amenazas son aquellas situaciones que provienen del entorno y que pueden llegar a atentar incluso contra la permanencia de la organización. Cuando los factores externos son negativos, se habla de considerar a las amenazas tomando como referencia el mercado, la competitividad, así como las tendencias políticas, sociales, económicas y tecnológicas.

En este mismo orden de ideas, para David (2008), el análisis externo consiste en definir a través de una auditoría externa, las oportunidades que podrían beneficiar a una empresa y las amenazas que deben evitarse. A tales efectos el objetivo de la auditoría externa es identificar las principales variables que ofrezcan respuestas prácticas. Las empresas deben responder a los factores de manera tanto ofensiva como defensiva, por medio de la formulación de estrategias que aprovechen las oportunidades externas o que reduzcan el impacto de las amenazas potenciales.

Según el mismo autor, para realizar el análisis externo deben evaluarse una serie de fuerzas claves como son: 1) fuerzas económicas; 2) fuerzas sociales, culturales, demográficas y ambientales; 3) fuerzas políticas, gubernamentales y legales; 4) fuerzas tecnológicas; y 5) fuerzas competitivas. De esta manera, las tendencias y los acontecimientos externos afectan de modo significativo a todos los productos, servicios, mercados y empresas del mundo.

El proceso de llevar a cabo una auditoría externa debe incluir a tantos gerentes $y$ empleados como sea posible. La participación en el proceso de dirección estratégica conduce a la comprensión y al compromiso de parte del personal de la empresa. Las personas aprecian la oportunidad de contribuir con ideas y obtener una mejor comprensión de la industria, los competidores y los mercados de su empresa.

Para realizar una auditoría externa, una empresa debe recabar primero información pertinente sobre las tendencias económicas, sociales, culturales, demográficas, ambientales, políticas, gubernamentales, legales y tecnológicas que le permita inferir las competitividades actuales. Por ello, el personal debe examinar varias fuentes de información, como revistas especiales y de negocios, así como periódicos. Este abordaje proporciona un flujo continuo de información estratégica oportuna y permite la participación de muchos empleados en el proceso de auditoría externa.

Los proveedores, distribuidores, vendedores, clientes y competidores representan otras fuentes de información vital. Una vez que se recaba la información, se debe asimilar y evaluar. De igual forma, se necesitan reuniones de gerentes para identificar, en forma conjunta, las oportunidades y las amenazas más importantes que enfrenta la empresa.

Otras variables que se utilizan con frecuencia son la participación en el mercado, la serie de productos en competencia, las economías mundiales, los socios extranjeros, la 
ventaja de las cuentas clave y de marca registrada, la competitividad en precios, los adelantos tecnológicos, la migración de las poblaciones, las tasas de interés y el control de la contaminación.

En este sentido, los factores externos clave deben ser: importantes para lograr objetivos y planes de acción, medibles, aplicables a todas las empresas competidoras y jerárquicos en el sentido de que algunos guardarán relación con la empresa en general y otros se centrarán más en áreas funcionales o de división. Una lista final de los factores externos más importantes se debe comunicar y distribuir en toda la empresa; tanto las oportunidades como las amenazas representan factores externos clave.

Así los factores económicos ejercen un impacto directo en el atractivo potencial de diversas estrategias; por ejemplo, cuando las tasas de interés aumentan, los fondos requeridos para la expansión de capital se vuelven más costosos o no están disponibles. Además, cuando las tasas de interés aumentan, el ingreso disponible disminuye y la demanda de productos que no son de primera necesidad se reduce. Cuando los precios de las acciones aumentan, se incrementa el deseo de adquirir acciones comunes como una fuente de capital para el desarrollo del mercado; además, cuando el mercado se expande, la riqueza de los consumidores y de las empresas crece.

De igual modo, los cambios sociales, culturales, demográficos y ambientales ejercen un impacto importante en casi todos los productos, servicios, mercados y clientes. Las oportunidades y amenazas que surgen de los cambios en las variables sociales, culturales, demográficas y ambientales impresionan y desafían a empresas grandes, pequeñas, lucrativas y no lucrativas de todas las industrias.

Respecto a las fuerzas políticas, gubernamentales y legales se tiene que los gobiernos federales, locales y extranjeros son los principales reguladores, liberalizadores, subsidiarios, patrones y clientes de las empresas; por lo tanto, los factores políticos, gubernamentales $y$ legales representan oportunidades o amenazas decisivas para las empresas tanto grandes como pequeñas.

Por otro lado, las fuerzas tecnológicas representan oportunidades y amenazas importantes que se deben tomar en cuenta al formular las estrategias. Los adelantos tecnológicos afectan en forma drástica los productos, servicios, mercados, proveedores, distribuidores, competidores, clientes, procesos de manufactura, prácticas de mercadotecnia y la posición competitiva de las empresas. Los adelantos tecnológicos crean nuevos mercados, dan como resultado la proliferación de productos nuevos y mejores, cambian las posiciones de los costos competitivos relativos en una industria y hacen que los productos y servicios existentes resulten obsoletos.

Así mismo, los cambios tecnológicos reducen o eliminan las barreras de costos entre las empresas, crean procesos de producción más cortos, producen escasez de habilidades técnicas y modifican los valores y las expectativas de los empleados, gerentes y clientes. Los adelantos tecnológicos generan nuevas ventajas competitivas que son más eficaces que las ventajas existentes. En la actualidad, ninguna empresa o industria se mantiene al margen de los adelantos tecnológicos que van surgiendo. 
Una parte fundamental de la auditoría externa son las fuerzas competitivas, que se refieren a identificar a las empresas rivales y determinar sus fortalezas, debilidades, capacidades, oportunidades, amenazas, objetivos y estrategias. La recolección y evaluación de información sobre los competidores es básica para la formulación de la estrategia con éxito. La identificación de los competidores no siempre es fácil ya que muchas empresas tienen divisiones que compiten en distintas industrias. Además, las empresas de propiedad privada no publican información financiera ni de mercadotecnia. No obstante, muchas empresas utilizan el Internet para obtener la mayor parte de su información sobre los competidores y llevar a cabo una auditoría externa.

De igual manera Serna (2008) coincide con las definiciones descritas con anterioridad sobre el análisis externo empresarial, y lo plantea como un factor determinante para enfrentar oportunamente en forma dinámica y acelerada los desafíos de los cambios, por ejemplo, los cambios de gustos de los clientes, de las condiciones políticas, de la estructura del mercado, así como los tecnológicos.

A este respecto, en el desarrollo de una estrategia, el medio se refiere a los factores que están fuera de la organización, ello incluye las fuerzas, los eventos y tendencias con los cuales la compañía interactúa. Generalmente, las fuerzas del medio se consideran fijas o dadas. No obstante, estas pueden ser influidas por la estrategia que se elija y tener un profundo impacto en el negocio.
Así, es necesario identificar las fuerzas importantes del medio, evaluarlas y hacerles seguimiento con el fin de que la dirección de la compañía pueda tomar un curso de acción efectivo. El examen del medio puede subdividirse en seis áreas clave, estas son: factores económicos, políticos, sociales, tecnológicos, competitivos y geográficos, cuyas definiciones guardan correspondencia con lo descrito anteriormente sobre estos factores.

Visto así, para el investigador si bien los factores y fuerzas del entorno generalmente se dan por hechos fuera del control de la firma, una vez situados y entendidos es posible desarrollar una estrategia que le permita reaccionar con antelación dichos factores. Así, la empresa podrá aprovechar sus oportunidades y anticipar el efecto de las amenazas que le genera su entorno, además, para diseñar estrategias que le permitan intervenir en los factores externos que la afecten.

\section{Visión}

Para David (2008), se debe responder a la pregunta básica: ¿qué se quiere llegar a ser? Una visión definida proporciona el fundamento para crear una declaración de la misión integral. Muchas empresas poseen tanto la declaración de la visión como la de la misión, pero la declaración de la visión debe establecerse en primer lugar. La declaración de la visión debe ser corta, formada de preferencia por una oración y desarrollada por tantos gerentes como sea posible. 
Según Serna (2008), la visión es un conjunto de ideas generales, algunas de ellas abstractas, que proveen el marco de referencia de lo que una empresa es y quiere ser en el futuro. La visión no se expresa en términos numéricos, la define la alta dirección de la compañía, debe ser amplia e inspiradora, conocida por todos e integrar al equipo gerencial a su alrededor, requiere líderes para su definición y para su cabal realización.

De la misma forma, la visión señala el rumbo, da dirección, sirve de guía en la formulación de las estrategias, a la vez que le proporciona un propósito a la organización. La visión debe reflejarse en la misión, los objetivos y las estrategias de la institución y se hace tangible cuando se materializa en proyectos y metas específicas, cuyos resultados deben ser medibles mediante un sistema de índices de gestión bien definidos. Por ello, el ejercicio de definir la visión es una tarea gerencial de mucha importancia, de allí que sea parte fundamental del proceso estratégico de una empresa.

A juicio del investigador, las distintas definiciones establecidas anteriormente acerca de la visión, guardan correspondencia entre sí, y sus fundamentos deben ser difundidos en la organización y reconocidos, por ello requiere un sistema de difusión que la haga conocer y comprender por todos los miembros de la organización.

\section{Misión}

Thompson y Strickland(2007) afirman que la misión es el motivo, propósito, fin o razón de ser de la existencia de una empresa u organización por que define: (1) lo que pretende cumplir en su entorno o sistema social en el que actúa, (2) lo que pretende hacer, y (3) él para quién lo va a hacer; y es influenciada en momentos concretos por algunos elementos como: la historia de la organización, las preferencias de la gerencia y /o de los propietarios, los factores externos o del entorno, los recursos disponibles, y sus capacidades distintivas.

Para Serna (2008) la misión implica una declaración única de cada organización porque sus principios, sus valores, su visión, la filosofía de sus dueños, los colaboradores y los grupos con los que interactúan en el mercado son para todas las organizaciones diferentes. La misión debe ser claramente formulada, difundida y conocida por todos los colaboradores de la organización para fomentar comportamientos cónsonos con la misma. Por consiguiente, la misión no puede convertirse en formulaciones que aparecen en las oficinas de la compañía. Sino que debe inducir comportamientos y crear compromisos.

De igual manera David (2008) sostiene que las declaraciones de la misión son: "expresiones perdurables de los propósitos que distinguen a una empresa de otras empresas similares. Una declaración de la misión identifica el alcance de las operaciones de una empresa en términos del producto y del mercado" (p.10). Aborda la pregunta básica que enfrentan todos los estrategas: "¿cuál es nuestro negocio?" La misión definida como una declaración describe los valores y las prioridades de una empresa.

Por ello, la elaboración de una declaración de la misión impulsa a los estrategas a considerar la naturaleza y el alcance de las 
operaciones actuales; y a evaluar el atractivo potencial de los mercados y las actividades en el futuro. Una declaración de la misión expresa en forma amplia la dirección de una empresa a futuro. La declaración de la misión, una declaración duradera sobre el propósito que distingue a una empresa de otra similar, es la declaración de la "razón de ser" de una empresa.

A su vez, una declaración de la misión definida es esencial para establecer objetivos y formular estrategias con eficacia. Una declaración de la misión, denominada en ocasiones una declaración del credo, una declaración del propósito, una declaración de la filosofía, una declaración de creencias, una declaración de principios de negocios o una declaración que define el negocio revela lo que una empresa desea ser y a quién quiere servir. La misión de una empresa es el fundamento de prioridades, estrategias, planes y tareas; es el punto de partida para el diseño de trabajos de gerencia $y$, sobre todo, para el diseño de estructuras de dirección. Nada podría parecer más sencillo o evidente que saber cuál es el negocio de una empresa.

Por tanto, la misión debe ser claramente formulada, difundida y conocida por todos los colaboradores. Los comportamientos de la organización deben ser consecuentes con esta misión, así como la conducta de todos los miembros de la organización. La misión de una empresa debe inducir comportamientos, crear compromisos. La vida de la organización tiene que ser consecuente con sus principios y valores, su visión y su misión.

En consideración a lo expuesto anteriormente, el autor de esta investigación estableció que debe existir una misión donde se manifiesten las actividades inherentes a las instituciones universitarias objeto de estudio. Finalmente es importante que quienes la gerencian se encarguen de su divulgación y su internalización por parte del personal.

\section{Objetivos}

David (2008) los define como resultados específicos que una empresa intenta lograr para cumplir con su misión básica. Los objetivos son indispensables para obtener el éxito de una empresa, debido a que establecen la dirección a seguir, ayudan en la evaluación, crean sinergia, revelan prioridades, enfocan la coordinación y proporcionan una base para llevar a cabo con eficacia las actividades de planificación, organización, motivación y control.

Agregan Thompson y Strickland (2007) que un objetivo de organización es el blanco hacia el que se orientan los esfuerzos que lleva a cabo una organización. Unos objetivos claros aportan fundamentos sólidos para la formulación de una estrategia, para la ejecución de la misma y para el planteamiento de la acción, por lo cual deben ser especificados y divulgados en las empresas.

En tal sentido, es conveniente que los objetivos sean: cuantitativos, cuantificables, realistas, comprensibles, desafiantes, jerárquicos, fáciles de lograr y congruentes entre las unidades de la empresa. Por tanto, cada objetivo debe relacionarse con un límite de tiempo. Los objetivos se establecen con frecuencia en términos como crecimiento en activos, crecimiento en ventas, rentabilidad, participación en el mercado, grado y naturaleza de diversificación, grado y naturaleza de integración vertical, ganancias por acción y responsabilidad social. 
Ahora bien, los objetivos establecidos con claridad ofrecen muchos beneficios, pues proporcionan dirección, permiten la sinergia, ayudan en la evaluación, establecen prioridades, reducen la incertidumbre, disminuyen al mínimo los conflictos, estimulan el desempeño y ayudan tanto en la distribución de recursos como en el diseño de trabajos. Los objetivos son necesarios en los niveles funcionales, de división y corporativos de una empresa, además constituyen una medida importante del desempeño de la gerencia.

De igual manera, los objetivos establecen las prioridades de la empresa; estimulan el desempeño y los logros; sirven como normas para evaluar a los individuos, grupos, departamentos, divisiones y empresas completas; proporcionan la base para el diseño de trabajos y las actividades de organización que se llevarán a cabo en una empresa; $y$, además, ofrecen dirección y permiten la sinergia en la empresa. Es difícil concebir a una empresa o individuo exitoso sin objetivos definidos, ya que el éxito ocurre en raras ocasiones por accidente; al contrario es el resultado del trabajo intenso dirigido hacia el logro de ciertos objetivos.

Por su parte Serna (2008), define los objetivos como "resultados a largo plazo que una organización espera lograr para hacer real la misión y la visión de la empresa o área de negocio" (p.73). Estos objetivos empresariales, junto con las estrategias, son los factores integradores de la tarea de la alta gerencia $y$, por tanto, deberán reflejarse en los planes funcionales y operativos de cada unidad estratégica de negocio.
Los planteamientos antes mencionados le permitieron al investigador considerar los objetivos en términos de propósitos a lograr, lo cual implica que existen coincidencias en sus apreciaciones. Cabe mencionar que tales objetivos deben ser revisados desde la perspectiva de David (2008), para verificar si los mismos se consideran en la actividad de las instituciones universitarias públicas de la Costa Oriental del Lago.

\section{Estrategias}

En el criterio de Mintzberg (2008), la estrategia comprende el conjunto de acciones integradas que definen un curso de acción o guía para enfrentar una situación. Por ello, explica que la estrategia debe ser definida a través de la integración y complementariedad de sus distintas acepciones: como plan, pauta, táctica, posición y perspectiva.

En el mismo orden de ideas para David (2008), "son los medios por los cuales se logran los objetivos a largo plazo" (p.12). Las estrategias de negocios incluyen la expansión geográfica, la diversificación, la adquisición, el desarrollo de productos, la penetración en el mercado, la reducción de costos, la enajenación, la liquidación y las empresas conjuntas.

Las estrategias son acciones potenciales que requieren decisiones de parte de la gerencia y de recursos de la empresa. Además, las estrategias afectan las finanzas a largo plazo de una empresa, por lo menos durante cinco años, orientándose así hacia el futuro. Las estrategias producen efectos en las funciones y divisiones de la empresa, y exigen que se tomen en cuenta tanto los factores externos 
como los factores internos que enfrenta la empresa.

En este sentido Serna (2008) argumenta que las estrategias son "las acciones que deben realizarse para mantener y soportar el logro de los objetivos de la organización y de cada unidad de trabajo y así hacer realidad los resultados esperados al definir los proyectos estratégicos" (p.73). Las estrategias son, entonces, las que permiten lograr y hacer realidad cada objetivo y cada proyecto estratégico.

En este sentido, diversas empresas buscan una combinación de estrategias en forma simultánea para el logro de los objetivos planteados. Por otro lado, difícilmente las empresas puedan aplicar un gran número de estrategias, por tanto, es necesario tomar decisiones y establecer prioridades evitando el endeudamiento excesivo. Las empresas no tienen la posibilidad de hacer adecuadamente muchas cosas porque los recursos y las habilidades se diluyen y los competidores obtienen ventaja.

En grandes organizaciones diversificadas se emplea con frecuencia una estrategia combinada cuando diferentes divisiones siguen diversas estrategias. Además, las empresas que luchan por sobrevivir deben emplear una combinación de distintas estrategias defensivas, como la enajenación, la liquidación y el recorte de gastos, en forma simultánea.

De igual forma, la integración hacia delante, la integración hacia atrás y la integración horizontal se conocen a menudo como estrategias de integración vertical. Las estrategias de integración vertical permiten a una empresa obtener control sobre distribuidores, proveedores y competidores.

Asimismo, la integración hacia delante implica la obtención de la propiedad o aumento del control sobre distribuidores o vendedores a minoristas. Gran cantidad de empresas de manufactura (proveedores) siguen una estrategia de integración hacia delante por medio del establecimiento de sitios electrónicos para vender en forma directa productos a los consumidores. Los costos relativamente bajos de las ventas en línea en comparación con los costos de las ventas en tiendas a minoristas convierte la integración hacia delante en una estrategia muy atractiva para muchos proveedores.

Un medio eficaz para implantar la integración hacia delante es la franquicia. Las empresas se expanden con rapidez por medio de la franquicia porque los costos y las oportunidades se diluyen entre muchos individuos.

Por otro lado, la integración hacia atrás es una estrategia que busca la obtención de la propiedad o el aumento del control sobre los proveedores de una empresa. Esta estrategia es apropiada cuando los proveedores actuales de una empresa son poco confiables, demasiado costosos o no satisfacen las necesidades de la empresa. La competencia global estimula a las empresas a reducir su número de proveedores y a exigir mayores niveles de servicio y calidad de aquellos que conserve.

Otra estrategia usada es la integración horizontal, que se refiere a la busca del aumento del control sobre los competidores de una empresa. Una de las tendencias más significativas en la dirección estratégica 
actual es el aumento del uso de la integración horizontal como una estrategia de crecimiento. Las fusiones, las adquisiciones y la toma de control entre competidores permiten el incremento de las economías de escala y el mejoramiento de la transferencia de recursos y capacidades.

Así mismo, se tienen las estrategias intensivas, que buscan la penetración en el mercado y el desarrollo de productos. En este sentido, una estrategia de penetración en el mercado intenta aumentar la participación de los productos o servicios presentes en los mercados actuales a través de mayores esfuerzos de mercadotecnia. La penetración en el mercado incluye el aumento en el número de vendedores, el incremento en los gastos de publicidad, la oferta de artículos de promoción de ventas en forma extensa y el aumento de los esfuerzos publicitarios.

De igual forma, el desarrollo de productos es una estrategia que intenta aumentar las ventas por medio del mejoramiento o la modificación de los productos o servicios actuales. El desarrollo de productos implica por lo general grandes gastos en investigación y desarrollo.

También, existen las estrategias de diversificación que son menos conocidas ya que las empresas encuentran más dificultades para manejar diversas actividades de negocios. Además de las estrategias de integración, intensivas y de diversificación, las empresas tienen también la posibilidad de utilizar el recorte de gastos, la enajenación o la liquidación.

Tomando en cuenta las definiciones anteriores, el investigador sostiene que a pesar de las diversas estrategias de acción existentes, lo más sustancial es que la empresa seleccione aquellas estrategias que más favorezcan el cumplimiento de la visión, misión y objetivos corporativos. Además, el investigador manifiesta que las instituciones universitarias públicas de la Costa Oriental del Lago deben formular y evaluar las estrategias ya que estas representan el medio para superar las debilidades, amenazas, y para aprovechar las oportunidades y fortalezas.

\section{MÉTODO}

La investigación es descriptiva con diseño no experimental, transeccional y de campo. La población quedó constituida por 51 personas encargadas de los procesos de admisión estudiantil de la Universidad del Zulia Núcleo Costa Oriental del Lago, Universidad Nacional Experimental "Rafael María Baralt y el Instituto de Tecnología de Cabimas.

La recolección de la información se llevó a cabo a través de un cuestionario autoadministrada conformado por 18 ítems con un escalamiento de frecuencia con cinco alternativas de respuestas, cuyas categorías fueron: Siempre (5); Casi siempre (4); Algunas veces (3); Casi nunca (2) y Nunca (1). El cuestionario cual fue validado por el juicio de seis (6) expertos y obtuvo un 0,97 de confiabilidad según el coeficiente Alfa Cronbach.

Una vez recolectados los datos se procedió al análisis de los resultados obtenidos, empleándose la estadística de tipo descriptiva, la cual permitió caracterizar cada uno de los indicadores que miden la planificación en los procesos de admisión estudiantil. 
Con respecto a la técnica de tendencia central, se utilizó la media aritmética. Para tal fin, el investigador diseñó un rango, sobre la base de la puntuación más alta y más baja de la escala de referencia utilizada, expresado de la siguiente manera: muy alta aplicación (4.21 - 5.00); alta aplicación (3.41 - 4.20); moderada aplicación (2.61 - 3.40); baja aplicación $(1.81-2.60)$ y muy baja aplicación (1.00 - 1.80).Seguidamente se obtuvo una matriz de datos, que permitió el análisis detallado de la variable objeto de estudio.

RESULTADOS

La Tabla 1 muestra el comportamiento de las respuestas suministradas por la unidades informantes en función del indicador análisis interno, arrojando una media de 4,16, mostrando una alta aplicación al momento de la recolección y asimilación de información sobre las operaciones inherentes a los procesos de admisión, permitiendo con ello determinar las fortalezas y debilidades más importantes de las instituciones analizadas.

Considerando los resultados de cada ítem, donde el indicador arriba a la categoría de alta aplicación, se deduce que se están validando los postulados teóricos. Así las cosas, existe una correspondencia entre el análisis interno realizado por quienes gestionan la logística en los procesos de admisión estudiantil aplicados por las instituciones universitarias públicas de la Costa Oriental del Lago y los postulados teóricos de Serna (2008), quien considera que el análisis interno parte del perfil de la capacidad interna de la organización $(\mathrm{PCl})$, el cual es un medio para evaluar las fortalezas y debilidades involucrando todos los factores que puedan afectar sus operaciones.

Tabla 1. Indicador: Análisis interno

\begin{tabular}{llcc}
\hline \multicolumn{4}{c}{ Ítems } \\
\hline 1 & La dirección evalúa los atributos de la institución que favorecen el logro de los objetivos. \\
2 & Se examinan las condiciones de la institución que dificultan el éxito del proceso de admisión \\
3 & $\begin{array}{l}\text { La institución conoce la capacidad tecnológica disponible para afrontar las exigencias del proceso } \\
\text { de admisión }\end{array}$ & $\mathbf{2}$ & $\mathbf{3}$ \\
ítems & $\mathbf{1}$ & 4,06 & 4,25 \\
$\overline{\boldsymbol{X}}$ & 4.18 & Alta aplicación & Muy alta aplicación \\
Categoría & Alta aplicación & $\mathbf{4 , 1 6}$ & \\
$\begin{array}{l}\text { Promedio } \boldsymbol{X} \\
\text { Categoría }\end{array}$ & Alta aplicación \\
\hline
\end{tabular}

Fuente: Elaboración propia.

De manera que, según los resultados obtenidos las instituciones bajo estudio cuentan con ciertas fortalezas que les permiten llevar a cabo los procesos de admisión, siendo una de ellas su capacidad tecnológica disponible.
Seguidamente, en la Tabla 2 se resume el comportamiento del indicador análisis externo, donde se observa una media de 3,98 para este indicador, implicando alta aplicación de la revisión de las variables que rodean el ambiente organizacional, incluyendo oportunidades y amenazas. 
Tabla 2. Indicador: Análisis externo

\begin{tabular}{llcc}
\hline \multicolumn{4}{c}{ Ítems } \\
\hline 4 & Se consideran las nuevas tecnologías del mercado que agilicen el proceso de admisión \\
5 & Se evalúan los factores políticos del entorno que pueden amenazar el rendimiento del proceso de \\
& admisión \\
6 & Se estiman los cambios en la economía global que afectan el proceso de admisión \\
Ítems & $\mathbf{4}$ & $\mathbf{5}$ & $\mathbf{6}$ \\
$\overline{\boldsymbol{X}}$ & 3,92 & 3,98 & 3,86 \\
Categoría & Alta aplicación & Alta aplicación & Alta aplicación \\
$\begin{array}{l}\text { Promedio } \boldsymbol{X} \\
\text { Categoría }\end{array}$ & $\mathbf{3 , 9 8}$ & \\
\hline
\end{tabular}

Fuente: Elaboración propia.

Como ha quedado de manifiesto, según los datos analizados las instituciones universitarias públicas bajo estudio, se enfrentan, al igual que las empresas, a retos importantes dentro del contexto donde se desarrollan, de manera que, deben definir a través de una auditoría externa, las oportunidades que podrían beneficiarla, así como las amenazas que deben evitarse. Al respecto, David (2008) expresa que el objetivo de la auditoría externa es identificar las principales variables que ofrezcan respuestas prácticas.

Ante este contexto teórico y en consonancia con los resultados descritos, se evidencia la evaluación de los factores tecnológicos, políticos y económicos, los cuales a criterio del investigador han cobrado relevancia estratégica e inciden al planificar los procesos de admisión.

En lo que respecta al indicador visión, se tiene una media de 3,92, tal como se muestra en la Tabla 3, indicando alta aplicación del indicador como parte del proceso de planificación en los procesos de admisión estudiantil aplicados por las instituciones universitarias públicas de la Costa Oriental del Lago.

Tabla 3. Indicador: Visión

\begin{tabular}{lccc}
\hline \multicolumn{4}{c}{ Ítems } \\
\hline 7 & La institución divulga al personal la visión logística \\
8 & El personal encargado del proceso de admisión conoce los principios de la visión. \\
9 & La institución busca el cumplimiento de lo establecido en su visión \\
\hline Ítems & $\mathbf{7}$ & $\mathbf{8}$ & $\mathbf{9}$ \\
\hline$\overline{\boldsymbol{X}}$ & 3,80 & 3,88 & 4,08 \\
Categoría & Alta aplicación & Alta aplicación & Alta aplicación \\
Promedio $\boldsymbol{X}$ & & $\mathbf{3 , 9 2}$ & \\
Categoría & & Alta aplicación & \\
\hline
\end{tabular}

Fuente: Elaboración propia. 
Estos resultados, reflejan que las instituciones universitarias analizadas a través de la visión busca anticiparse a las necesidades y deseos del cliente, gestionando la adquisición de capital, materiales, personas, tecnologías e información necesarias para lograr optimizar los procesos de admisión estudiantil aplicados por las instituciones universitarias públicas de la Costa Oriental del Lago.

Dado los resultados mostrados, se evidencia alta congruencia con lo planteado por Serna (2008), para quien la visión es un conjunto de ideas generales, algunas de ellas abstractas, que proveen el marco de referencia de lo que una empresa es y quiere ser en el futuro.

En la Tabla 4, mostrada para analizar el comportamiento del indicador misión, se evidencia una media de 3,78 indicando alta aplicación del motivo, propósito, fin o razón de ser de la existencia de las instituciones universitarias analizadas.

Tabla 4. Indicador Misión

\begin{tabular}{llcc}
\hline \multicolumn{4}{c}{ Ítems } \\
\hline 10 & Se tiene una declaración de la misión donde manifieste el propósito de la gestión logística \\
11 & La misión logística es conocida por el personal del proceso de admisión \\
12 & El comportamiento del personal encargado del & proceso de admisión es consecuente con lo \\
establecido en la misión & & $\mathbf{1 2}$ \\
Ítems & $\mathbf{1 0}$ & 3,86 & 3,78 \\
$\overline{\boldsymbol{X}}$ & 3,71 & Alta aplicación & Alta aplicación \\
Categoría & Alta aplicación & $\mathbf{3 , 7 8}$ & \\
$\begin{array}{l}\text { Promedio } \boldsymbol{X} \\
\text { Categoría }\end{array}$ & Alta aplicación & \\
\hline
\end{tabular}

Fuente: Elaboración propia.

La situación mostrada pone en evidencia que las instituciones bajo estudio tratan a través de la misión fomentar comportamientos cónsonos con los procesos de admisión estudiantil aplicados por las instituciones universitarias públicas de la Costa Oriental del Lago, identificando el alcance de las actividades necesarias.
Estos resultados, al reflejar alta aplicación, pone de manifiesto alta coincidencia con lo expresado por Thompson y Strickland (2007) para quien la misión es la razón de ser de la existencia de una empresa por que define lo que pretende cumplir en su entorno o sistema social en el que actúa, lo que pretende hacer, y él para quién lo va a hacer. 
Tabla 5. Indicador: Objetivos

\begin{tabular}{|c|c|c|c|}
\hline \multicolumn{4}{|c|}{ Ítems } \\
\hline \multicolumn{4}{|c|}{ La institución establece objetivos medibles para el proceso logístico } \\
\hline \multicolumn{4}{|c|}{ Los objetivos trazados reflejan las prioridades en cuanto a logística } \\
\hline \multicolumn{4}{|c|}{ El personal encargado del proceso logístico busca el logro de los objetivos } \\
\hline Ítems & 13 & 14 & 15 \\
\hline $\bar{X}$ & 3,78 & 4,00 & 4,25 \\
\hline Categoría & Alta aplicación & Alta aplicación & Muy alta aplicación \\
\hline \multicolumn{2}{|l|}{ Promedio $X$} & 4,01 & \\
\hline \multicolumn{2}{|l|}{ Categoría } & Alta aplicación & \\
\hline
\end{tabular}

Fuente: Elaboración propia.

Ahora bien, en el orden de presentación de los resultados, se tienen los valores para el indicador objetivos. En este sentido, en la Tabla 5, se observa un promedio de 4,01 indicando según las respuestas dadas por los encuestados, una alta aplicación del fin último al que se dirige las acciones necesarias para lograr el proceso de planificación en los procesos de admisión estudiantil.

Los resultados demuestran que en las instituciones analizadas, evaluar los logros, medir el progreso o detectar fallos o desviaciones son algunos de los aspectos claves establecidos en sus objetivos a fin de lograr una adecuada planificación en sus procesos de admisión. Esta situación, de alta aplicación del indicador, valida la posición teórica planteada por Serna (2008), cuando indica que los objetivos son los resultados que una organización espera lograr para hacer real su misión y la visión.
Para cerrar el análisis de la dimensión proceso de planificación, se presentan los hallazgos obtenidos para el indicador denominada estrategias. Para ello, se muestra la Tabla 6, donde se recogen los resultados del indicador con un promedio de 4,09 indicando según las respuestas dadas por los encuestados, una alta aplicación de un conjunto de acciones integradas que definen los cursos de acción del proceso de planificación en los procesos de admisión estudiantil.

Según las evidencias anteriores, en las instituciones analizadas se consideran las estrategias para alcanzar el proceso de planificación en los procesos de admisión estudiantil aplicados por las instituciones universitarias públicas de la Costa Oriental del Lago, para ello buscan una combinación de estrategias en forma simultánea para el logro de los objetivos planteados. 
Tabla 6. Indicador Estrategias

\begin{tabular}{|c|c|c|c|}
\hline \multicolumn{4}{|c|}{ Ítems } \\
\hline \multicolumn{4}{|c|}{ Se utiliza la información de los análisis del entorno para formular estrategias } \\
\hline \multicolumn{4}{|c|}{ Las estrategias que se establecen buscan cumplir los objetivos logísticos } \\
\hline \multicolumn{4}{|c|}{ La institución determina de qué manera se asignan los recursos para cumplir los objetivos. } \\
\hline Ítems & 16 & 17 & 18 \\
\hline$\overline{\boldsymbol{X}}$ & 3,96 & 4,39 & 3,92 \\
\hline Categoría & Alta aplicación & Muy alta aplicación & Alta aplicación \\
\hline Promedio $X$ & & 4,09 & \\
\hline Categoría & & Alta aplicación & \\
\hline
\end{tabular}

Fuente: Elaboración propia.

Así entonces, los resultados sobre las estrategias en la gestión logística bajo análisis, muestran alta aplicación, lo cual coincide con Serna (2008), quien argumenta que las estrategias son acciones que deben realizarse para mantener y soportar el logro de los objetivos de la organización y de cada unidad de trabajo para hacer realidad los resultados esperados al definir los proyectos estratégicos. De tal manera, las estrategias permiten lograr y hacer realidad cada objetivo y cada proyecto estratégico.

Para la dimensión proceso de planificación, cuyos resultados se concentran en la tabla 7 , se observa un promedio de 3,89, indicando alta aplicación de este proceso de planificación que se desarrolla en los procesos de admisión estudiantil aplicados por las instituciones universitarias públicas de la Costa Oriental del Lago.

Tabla 7. Dimensión: Proceso de planificación

\begin{tabular}{ccl}
\hline Indicador & $X$ & Categoría \\
\hline Análisis interno & 4,16 & Alta aplicación \\
Análisis externo & 3,98 & Alta aplicación \\
Visión & 3,92 & Alta aplicación \\
Misión & 3,78 & Alta aplicación \\
Objetivos & 4,01 & Alta aplicación \\
Estrategias & 4,09 & Alta aplicación \\
Promedio & 3,99 & Alta aplicación
\end{tabular}

Fuente: Elaboración propia.

Dentro de este contexto, se refleja que en las instituciones analizadas el proceso de la planificación tiene el propósito de preparar y adecuar las bases ante los cambios repentinos del mercado. Para ello hay que orientar las funciones hacia los nuevos retos y exigencias que se originen. $Y$ una vez definida la estructura es importante establecer políticas y reglas que guíen el comportamiento a nivel individual y grupal del personal de las instituciones universitarias analizadas.

Al ver los resultados se evidencia alta congruencia con la teoría manejada, en lo que respecta a lo planteado por Sople (2007), para quien la planificación es aquella 
parte de la gestión logística que se encarga de definir los recursos necesarios para cada función; así como las áreas de atención y de mejoras para lograr los objetivos generales, lo cual indudablemente repercutirá en el rendimiento y en el grado de satisfacción de los clientes.

Aun cuando este proceso de planificación fue catalogado con alta aplicación, el mismo no alcanzó la categoría máxima de análisis (muy alta aplicación), por lo que es importante que quienes gestionan los procesos de admisión, consideren la planificación, como una herramienta administrativa que ayuda a incrementar las posibilidades de éxito.

\section{CONCLUSIONES}

Una vez analizada la planificación en los procesos de admisión estudiantil de las instituciones universitarias públicas de la Costa Oriental del Lago, se concluye una alta aplicación de la planificación como un proceso a través del cual se establecen los cursos de acción adecuados para alcanzar los objetivos trazados en los procesos de admisión analizados.

Al detalle se observó alta aplicación al momento de la recolección y asimilación de información sobre las operaciones inherentes a los procesos de admisión (análisis interno); revisión de las variables que rodean el ambiente organizacional, incluyendo oportunidades y amenazas (externo), anticiparse a las necesidades y deseos del cliente (visión), razón de ser de la existencia de las instituciones universitarias analizadas (misión), dirección de las acciones necesarias para lograr el proceso de planificación (objetivos) y de un conjunto de acciones integradas que definen los cursos de acción (estrategias) del proceso de planificación de la gestión logística en los procesos de admisión estudiantil.

\section{REFERENCIAS}

Chiavenato, I. (2007). Administración en los nuevos tiempos. México: Editorial Limusa, S.A

David, F. (2008). Conceptos de administración estratégica. 11a ed. México: Ed. Pearson Prentice Hall

Hellriegel, S.; Jackson, J. y Slocum, W. (2005). Administración: un enfoque basado en competencias. 10ma ed. México: Ed. Thomson

Hurtado, D. (2008). Principios de administración. Primera edición. Fondo editorial ITM Medellín, Colombia

Mintzberg, H. (2008).El Proceso Estratégico. México: Editorial Pearson Educación

Serna, H. (2008). Gerencia Estratégica. 10a Edición. Bogotá: Editorial 3R

Sople, V. (2007). Logistics management: the supply chain imperative. India: Ed. Pearson education

Stoner J., y Wankel, C., (2005) Administración, Edición: $5^{a}$ ed., México: Editorial: PrenticeHall

Thompson, A. y Strickland, A. (2007). Administración Estratégica. Textos y Casos. $13^{\circ}$ Edición. Bogotá: McGraw Hill 